\title{
Comment on "A centroid molecular dynamics study of liquid para hydrogen and ortho deuterium" [J. Chem. Phys. 121, 6412 (2004)]
}

\author{
Thomas F. Miller III, David E. Manolopoulos and Paul A. Madden \\ Physical and Theoretical Chemistry Laboratory, Oxford University, South Parks Road, Oxford OX1 3QZ, \\ United Kingdom \\ Martin Konieczny \\ Institut für Theoretische Physik II, Heinrich-Heine-Universität Düsseldorf, Universitätsstraße 1, \\ D-40225 Düsseldorf, Germany \\ Harald Oberhofer \\ Faculty of Physics, University of Vienna, Boltzmanngasse 5, A-1090 Vienna, Austria
}

(Received 11 October 2004; accepted 5 November 2004; published online 18 January 2005)

[DOI: $10.1063 / 1.1839867]$

Hone and Voth ${ }^{1}$ have recently reported both classical and centroid molecular dynamics (CMD) simulations of liquid para hydrogen at two different state points $(T=25 \mathrm{~K}, \rho$ $=0.0190 \AA^{-3}$ and $T=14 \mathrm{~K}, \rho=0.0235 \AA^{-3}$ ), and of ortho deuterium at one $\left(T=20.7 \mathrm{~K}, \rho=0.0254 \AA^{-3}\right)$. A striking result of their study was that quantum mechanical effects were found to increase the diffusion in liquid para hydrogen, but to decrease it in ortho deuterium. ${ }^{1}$ Here we provide the explanation for this result.

In search of this explanation, we have investigated the system-size dependence of the classically computed diffusion constants. Our simulations were performed at the same three thermodynamic state points, and used the same isotropic $\left(\right.$ Silvera-Goldman ${ }^{2}$ ) pair potential as Hone and Voth for the interaction between $J=0$ hydrogen (deuterium) molecules. In each $(N V E)$ simulation, we equilibrated the system for $20 \mathrm{ps}$, and then calculated the velocity autocorrelation function for 2 ps by averaging over 100 consecutive 4 ps trajectories with a time step of $0.5 \mathrm{fs}$. The temperature was controlled by resampling momenta from the Maxwell distribution between each trajectory. This procedure was repeated five times to obtain an average value for (and a standard error in) the diffusion constant of each simulation.

The resulting diffusion constants are compared with those of Hone and Voth ${ }^{1}$ in Table I. It can be seen from this table that our classical results agree well with theirs for the one classical system size (343 molecules) that they considered. However, it is also clear that, while it suffices for ortho deuterium, this system is too small to give a converged result for para hydrogen. The reason for this becomes apparent when one considers Fig. 1, which shows a typical configuration from our simulation at the $25 \mathrm{~K}$ state point of a system with 500 molecules $\left(5^{3}\right.$ face-centered cubic unit cells). A large bubble has formed in the middle of the simulation cell, and we have found that a similar bubble also forms at the 14 $\mathrm{K}$ state point. The spontaneous formation of these bubbles is a clear indication that both state points lie in the liquid-vapor coexistence region of the classical phase diagram.

The densities that Hone and Voth chose for their two para hydrogen state points were obtained from earlier isothermal-isobaric path integral Monte Carlo calculations at zero pressure. ${ }^{3}$ These quantum mechanical densities are about $2 / 3$ of the zero-pressure liquid densities obtained in purely classical Monte Carlo simulations. ${ }^{3}$ It is not therefore surprising that these state points lie in the classical liquidvapor coexistence region and lead to phase separation.

The effect of the phase separation on the calculated diffusion constant can be understood from the following argument. The diffusion of the molecules at the surface of the bubble is faster than that of the molecules in the bulk liquid, because the surface of the bubble has a lower density. Since the fraction of molecules at the surface is proportional to $N^{-1 / 3}$, where $N$ is the number of molecules in the simulation cell, one would expect the calculated diffusion constant to satisfy

$$
D(N)=D_{\infty}+b N^{-1 / 3},
$$

where $D_{\infty}$ is the diffusion constant in the high-density liquid phase and $b$ is a positive constant.

This expectation is confirmed in Fig. 2, which plots the classical data for para hydrogen in Table I as a function of $N^{-1 / 3}$. The resulting values of $D_{\infty}$ are $0.56(2) \AA^{2} / \mathrm{ps}$ at the $25 \mathrm{~K}$ state point and $0.02(1) \AA^{2} /$ ps at $14 \mathrm{~K}$, where the numbers in parentheses are the standard errors in the last digit as obtained from linear regression. To the extent that one accepts the extrapolations to $N^{-1 / 3}=0$ in Fig. 2, these values are the correct classical molecular dynamics results for the two para hydrogen state points. [For reference, earlier classical molecular dynamics simulations at densities corresponding to zero external pressure have been found to give $D=0.5$ and $0 \AA^{2} / \mathrm{ps}$ (solid) at 25 and $14 \mathrm{~K}$, respectively. ${ }^{4}$ Strictly speaking, the pressure at which $D_{\infty}$ is obtained from the extrapolations in Fig. 2 is not zero, but rather the classical liquid vapor pressure at each temperature.]

The implications of all this for quantum diffusion in liquid para hydrogen and ortho deuterium are as follows. The classical and quantum CMD results for para hydrogen in the paper by Hone and Voth ${ }^{1}$ pertain to different situations. The quantum fluid is in the liquid phase at both para hydrogen state points, whereas the classical fluid is in the liquid-vapor 
TABLE I. Calculated diffusion constants for para hydrogen and ortho deuterium.

\begin{tabular}{lcccc}
\hline \hline & & \multicolumn{3}{c}{ Diffusion constant $\left(\AA^{2} / \mathrm{ps}\right)^{\mathrm{b}}$} \\
\cline { 3 - 5 } Calculation $^{\mathrm{a}}$ & System size & $\mathrm{H}_{2}(25 \mathrm{~K})$ & $\mathrm{H}_{2}(14 \mathrm{~K})$ & $\mathrm{D}_{2}(20.7 \mathrm{~K})$ \\
\hline Hone and Voth & & & & \\
CMD & 180 & $1.52(8)$ & $0.35(5)$ & $0.40(6)$ \\
Classical & 343 & $1.20(6)$ & $0.18(2)$ & $0.52(4)$ \\
Present work & & & & \\
hcp $4\left[5 \times 3^{2}\right]$ & 180 & $1.40(1)$ & $0.230(2)$ & $0.500(2)$ \\
fcc $4\left[4^{3}\right]$ & 256 & $1.31(1)$ & $0.206(2)$ & $0.500(2)$ \\
sc $1\left[7^{3}\right]$ & 343 & $1.23(1)$ & $0.183(2)$ & $\ldots$ \\
fcc $4\left[5^{3}\right]$ & 500 & $1.16(1)$ & $0.168(2)$ & $\ldots$ \\
fcc $4\left[6^{3}\right]$ & 864 & $1.06(1)$ & $0.145(2)$ & $\cdots$ \\
\hline \hline
\end{tabular}

${ }^{a}$ The CMD and classical results of Hone and Voth are taken from Ref. 1.

${ }^{\mathrm{b}}$ The number in parentheses after each entry is the standard error in the last digit. Note that the present ortho deuterium results are already well converged with a system size of 180 molecules.

coexistence region. This does have an indirect effect on the diffusion coefficient, but it is a rather artificial one. The classical fluid phase separates into a low-density vapor region and a high-density liquid region, the latter of which determines the diffusion constant as $N \rightarrow \infty$. Since this liquid region has a significantly higher density than the corresponding quantum liquid, the classical diffusion constant is found to be lower, but then this involves a comparison between two very dissimilar systems.

On the other hand, it is clear from the system-size independence of our classical results for ortho deuterium in Table I that the state point considered by Hone and Voth for this molecule is in the stable branch of the classical liquid. In this

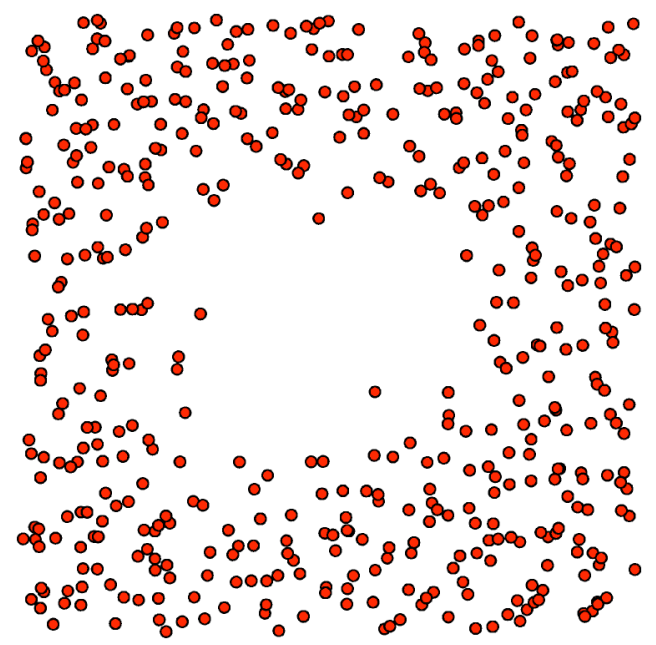

FIG. 1. A typical configuration from our 500 molecule classical molecular dynamics simulation of para hydrogen at the $T=25 \mathrm{~K}$ and $\rho$ $=0.0190 \AA^{-3}$ state point. Note the bubble.
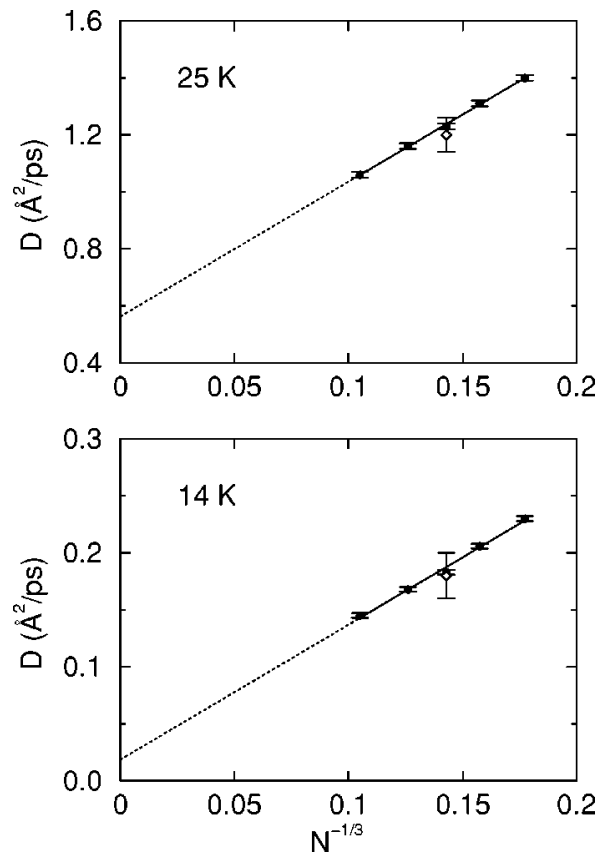

FIG. 2. System-size scaling of our computed classical diffusion constants for para hydrogen (filled circles) at the $T=25 \mathrm{~K}, \rho=0.0190 \AA^{-3}$ and $T$ $=14 \mathrm{~K}, \rho=0.0235 \AA^{-3}$ state points. The empty diamonds are the results reported by Hone and Voth (Ref. 1).

case, the comparison between CMD and classical molecular dynamics does provide a valid way to assess the importance of quantum mechanical effects in liquid diffusion, and one sees from Table I that these effects lead to a reduction in the diffusion constant. This is consistent with the fact that quantum fluctuations cause molecules to "swell," as was illustrated for a hard sphere fluid by Chandler and Wolynes. When comparing quantum and classical systems with the same (uniform) number density, one would clearly expect the swollen molecules of the quantum system to diffuse more slowly.

Finally, we note that diffusion constants at the present three state points calculated using the ring polymer molecular dynamics method ${ }^{6}$ corroborate the CMD results of Hone and Voth and actually increase slightly rather than decrease when the system size is increased beyond 180 molecules. ${ }^{7}$ This indicates that all three state points do indeed lie in the quantum liquid region, as we have assumed throughout our discussion.

${ }^{1}$ T. D. Hone and G. A. Voth, J. Chem. Phys. 121, 6412 (2004).

${ }^{2}$ I. F. Silvera and V. V. Goldman, J. Chem. Phys. 69, 4209 (1978).

${ }^{3}$ D. Scharf, G. J. Martyna, and M. L. Klein, Low Temp. Phys. 19, 364 (1993).

${ }^{4}$ J. Cao and G. J. Martyna, J. Chem. Phys. 104, 2028 (1996).

${ }^{5}$ D. Chandler and P. G. Wolynes, J. Chem. Phys. 74, 4078 (1981).

${ }^{6}$ I. R. Craig and D. E. Manolopoulos, J. Chem. Phys. 121, 3368 (2004).

${ }^{7}$ T. F. Miller III and D. E. Manolopoulos, J. Chem. Phys. (to be published). 